\title{
A Framework for Electroencephalogram Process at Real-Time using Brainwave
}

\author{
Yunsick Sung ${ }^{\dagger}$, Kyungeun $\mathrm{Cho}^{++}$, Kyhyun $\mathrm{Um}^{+++}$
}

ABSTRACT

Neurofeedback training using ElectroEncephaloGrams (EEGs) is commonly utilized in the treatment of Alzheimer's disease, and Attention Deficit Hyperactivity Disorder (ADHD). Recently, BCI (Brain-computer Interface) contents have developed, not for the purpose of treatment, but for concentration improvement or brain relaxation training. However, as each user has different wave forms, it is hard to develop contents controlled by such different wave. Therefore, an EEG process that allows the ability to transform the variety of wave forms into one standard signal and use it without taking a user's characteristic of EEG into account, is required. In this paper, a framework that can reduce users' characteristics by normalizing and converting measured EEGs is proposed for contents. This framework also contains the process that controls different brainwave measuring devices. In experiment a handling process applying the proposed framework to the developed BCI contents is introduced.

Key words: Brainwave, BCI, Brain- ${ }^{-}$computer Interface, Electroencephalograms

\section{INTRODUCTION}

A brainwave is a wave formed measured by placing electrodes on the scalp nearby frontal lobe [1]. Brainwave measurements are usually used for the treatment or prevention of Alzheimer's disease and Attention Deficit Hyperactivity Disorder (ADHD). The Brain-computer Interface (BCI) $\mathrm{de}^{-}$

※ Corresponding Author: Kyungeun Cho, Address: Dept. of Multimedia Engineering, College of Digital Image and Contents, Dongguk University, 26, Pil-dong 3-ga jung-gu Seoul Korea 100-715, TEL : +82-2-2260-3834, FAX : +82-2-2260-3766, E-mail : cke@dongguk.edu Receipt date: Jan. 27, 2011, Revision date: June 14, 2011 Approval date: July 25, 2011

${ }^{+}$Dept. of Game Engineering, Graduate School of Dongguk University

(E-mail: sung@dongguk.edu)

${ }^{++}$Dept. of Multimedia Engineering, Dongguk University

${ }^{+++}$Dept. of Multimedia Engineering, Dongguk University

(E-mail:khum@dongguk.edu)

※ This paper is presented by summarizing part of the research, "A Development of Serious Game Technology using Brain Computer Interface" that was performed by Dongguk University and was supported by Korea Creative Content Agency(KOCCA) in the Culture Technology (CT) Research \& Developement Program 2009. vices to measure brainwaves are disseminated with high price. However, recently BCI device for contents has been released with relatively cheap price. Therefore users can easily use a BCI measuring device. On this account, various multimedia contents such as games using a BCI device have been developed.

The contents utilizing brainwaves can be div $^{-}$ ided into two types. One is neurofeedback [2]. It uses brainwaves that reflects user's characteristics, and is a form of training to make the desired brain status by adjusting brainwaves measured in real-time. The subject adjusts the development status of his disordered brain due to stress by conducting self-adjustment on brainwaves. Generally, the development of contents for treatment and prevention is based thereon.

The second type is contents such as a BCI game to maximize brainwave training. Usually, games are developed by using an interface such as a mouse, keyboard, pad, etc. Recently, while the studies of BCI have become reinforced, developments using brainwaves have been performed in 
games. For instance, BCI games are developed by generating concentration value and meditation value with measured brainwaves, then utilizing such values. Also, there is the application that extracts emotion status with measured brainwaves and utilizes it [3].

There are many attempts to develop BCI contents. Following features shall be provided to develop BCI contents conveniently. The first feature is a method that considers the varied amplitudes and number of vibrations of measured brainwaves, and provides standard signals for BCI contents. It costs a great deal of both time and money to develop BCI contents considering different brainwaves of users. Therefore, a process that can handle the variety of brainwaves of its users in BCI games is required. The second feature is the aspect of interfacing among brainwave measuring devices with an identical manner. Development including analysis of the various interfaces of brainwave devices also creates a significant issue in terms of cost and development time. In addition, implementing the required interface whenever there is a new brainwave measuring device is not easy.

This paper proposes an approach that can reduce users' characteristics by normalizing measured brainwaves. The brainwaves extracted from various users would be changed to standardized wave forms when the brainwaves are measured. Therefore applying them to a game would be easier. Additionally, the interface that can efficiently manage various brainwave measuring devices is introduced.

This paper is composed as follows. In chapter 2, we will describe studies related to the method applying brainwaves to BCI contents. In chapter 3 , the framework to conveniently use brainwaves in contents will be introduced. In chapter 4, we will introduce some cases applying the framework to BCI games. Finally, the conclusion for the proposed methods will be made in chapter 5 .

\section{RELATED WORKS}

While the price of brainwave measuring devices has been decreasing, the application range of $\mathrm{BCI}$ has been broadening. In particular, with the launch of brainwave measuring devices applicable to $\mathrm{BCI}$ contents development, studies to apply such devices are actively in progress [4].

P. A. Pour presents a brain data recording/ controlling system to control video games on mobile devices using electroencephalograph [5]. He processed brain data on a computer separate from the video game console, which has low delay in control signals. A. Nijholt surveys the BCI application in games and entertainment together with multiple possible input modalities such as a keyboard, speech, gestures, etc [6]. Andrea Finke presents a linear feature selection and classification scheme in BCI games to identify P300 events and calculate neurofeedback features [7]. E.C. Lalor presents an effective BCI application based ElectroEncephaloGrams (EEGs) for binary control in a real-time 3D game [8]. P. Martinez proposes a multistage procedure for a real-time BCI to allow users to navigate a small object in a virtual environment over time [9].

When a user plays games, the device does not always fit him effectively. Therefore, a learning process is necessary to find effective cooperation between a user and a BCI device. Beom-Soo Shim develops learning devices for balanced brain waves and tests in 3D games to increase the reliability and stability of gathered brain wave activity [10] K. Müller discusses machine learning methods and application of BCI for feature selection [11]. He points out that learning algorithms can help result in correct classifiers by offline testing. A.E. Selim also demonstrates that some machine learning algorithms may be applied for classification accuracy, including Bayesian Linear Discriminant Analysis (BLDA), linear Support Vector, Machine (SVM), Fisher Linear Discriminant Analysis, 
Generalized Anderson's Task linear classifier, and Linear Discriminant Analysis [12]. He finds that BLDA and SVM yielded the highest accuracy.

To reduce training time and improve speed of BCI performance, Yuanqing Li introduces a new semi-supervised support vector machine learning algorithm for BCI systems by joint feature $\mathrm{ex}^{-}$ traction and classification in iterations [13]. G. Schalk introduces and validates signal detection without analysis procedures [14]. He detects signals with Gaussian mixture models and implements in a package called SIGFRIED (SIGnal modeling For Realtime Identification and Event Detection).

Various studies are in progress to apply BCI to contents; among such, we will introduce a framework that can efficiently process EGGs in contents development in this paper.

\section{EEG FRAMEWORK}

In order to use the EEG signal in contents, measuring the EEG and changing it to be applicable for BCI contents are both required. In this paper, the EEG framework used to process and apply an EEG signal is proposed.

The EEG framework receives the input of brainwave values measured by using a BCI device as shown in Figure 1, and changes them to a signal

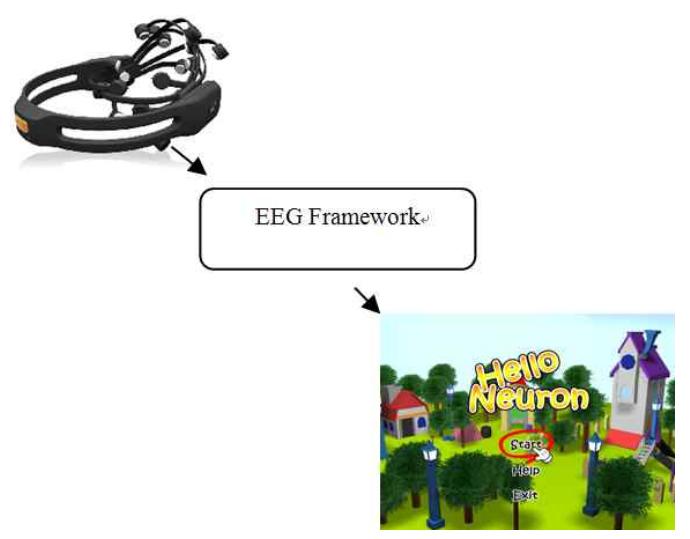

Fig. 1. EEG Flow Diagram suitable to BCI contents.

Brainwave handling processes are divided into three stages as shown in Figure 2. The first stage is to measure a signal from a $\mathrm{BCI}$ device and $\mathrm{pro}^{-}$ vide brainwaves so that other stages can utilize not only the measured raw data of brainwaves but also abstract values such as concentration and meditation. This stage provides a method that measures brainwaves through a common interface..

As shown in Figure 3, different BCI devices transfer brainwaves to the next stage through the brainwave receiving stage. Identical interface, $\mathrm{re}^{-}$ gardless of the type of brainwave device used, is available using EEG framework.

The second stage is to normalize measured brainwaves. This stage is divided into the brainwave collection step and the brainwaves change step. In the brainwave collection step, brainwaves are measured and collected from each user over a certain period, and occurrence frequency are calculated by counting frequency by section. During this step, measured brainwaves are not transferred to BCI contents.

In the third step, brainwaves to be applied to $\mathrm{BCI}$ contents are calculated in real-time by applying sectional occurrence frequencies to formula (1).

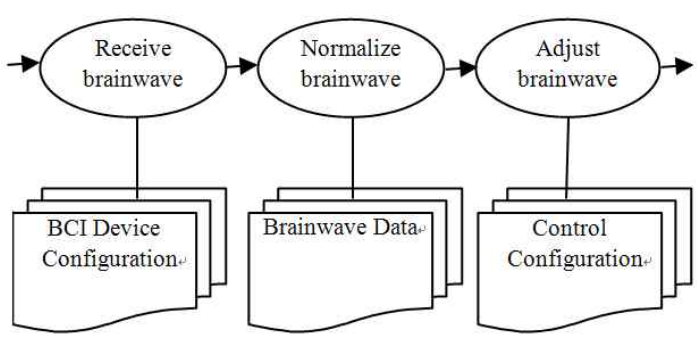

Fig. 2. EEG Framework

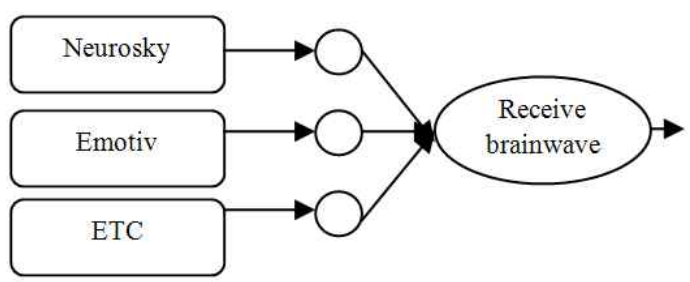

Fig. 3. $\mathrm{BCl}$ device connection 


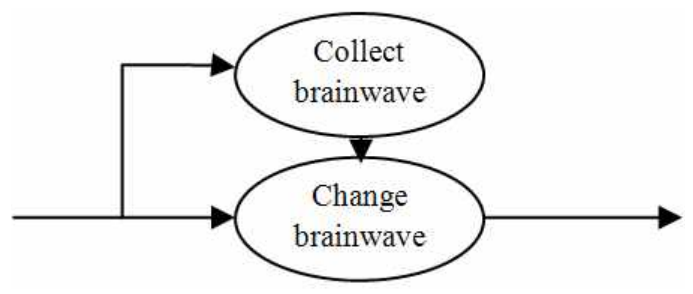

Fig. 4. EEG Normalization

Each brainwave is calculated by adding the relative value of collected brainwaves with the rate of brainwave $\mathrm{x}^{\prime}$ to the initial value $\mathrm{r}_{1}$ of the EEG range. Frequency $c_{m}$ has different values depending on the collected brainwaves. Therefore converting EEGs using formula (1) results in different distributions by user.

$$
y=r_{1}+\frac{\left(\sum_{l=r_{1}}^{x^{\prime}-1} c_{l}+\sum_{l=r_{1}}^{x^{\prime}} c_{l}\right) / 2}{\sum_{m=r_{1}}^{r_{k}} c_{m}} *\left(r_{k}-r_{1}\right)
$$

In the final stage users' brainwaves are amplified or shifted. Typically, various users' brainwaves are converted to similar signals through normalization, but it is hard to generate users' brainwaves in regular form when they are measured differently. On that account, normalized brainwave values are adjusted according to user. The amount of adjustment of brainwaves is predefined by each user. Normalized signals are changed again by formula 2 . The value $\mathrm{i}$ is used to increase or decrease the value $y$ and the value $r$ is used to amplified or reduced the value $(y+i)$.

$$
\mathrm{y}^{\prime}=(\mathrm{y}+\mathrm{i}) \times \mathrm{r}
$$

The signals that have been calculated through the last stage are transferred to BCI contents. The proposed framework processes EEGs with three divided stages: brainwave receiving, brainwave normalization, and brainwave adjustment. However, any stage may be eliminated or the order may be changed according to the characteristics of $\mathrm{BCI}$ contents.

\section{EXPERIMENT}

The Neurosky Mindset [15] is used in this experiment as a brainwave measuring device. It provides with raw data like the value of alpha wave, the value of beta wave and so on. It also provides concentration value and meditation value that are generated with combined raw data. Each value ranges from 0 to 100 .

Hello Neuron, an English word-learning game using $\mathrm{BCI}$, is utilized in the experiment. Using concentration values and meditation values, this game is composed to memorize English words in step by step.

Figure 5(a) shows the screen for memorizing an English word. When a user puts on a BCI device, the gauge shows the level of concentration on the

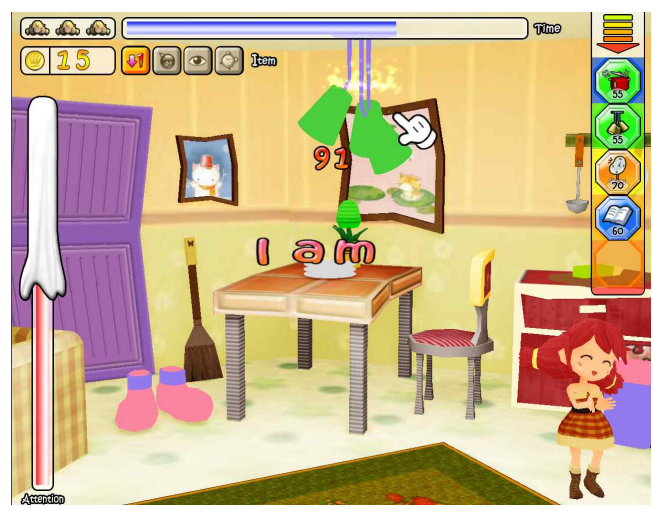

(a) English word memorization screen

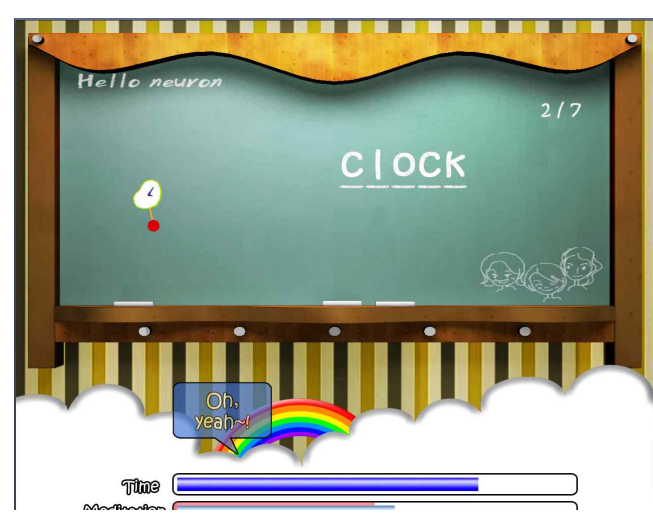

(b) English word review screen

Fig. 5. Hello Neuron 
left side of screen increases. The value of gauge ranges from 0 to 100. At first he selects the object in the list on the right side of screen and then find and click it in the 3D house. Secondly he concentrates his mind. The gauge will increase according to the level of his concentration. If the gauge reaches 60 , the name of the object will be appeared and is saved for the review. We decides the reaching value through experiments. Within 3 minutes, he should find all object memorizing its name.

The screen in Figure 5(b) shows the process to review memorized words. User relearns memorized words in the memorization phase. In this process, the opportunity is offered to answer the question of memorized words when the measured meditation value reaches 60 .

If Hello Neuron uses the measured brainwave directy, it would be hard to balance the level of learning difficulty, because of difference of brainwave

In order to apply the proposed framework for normalization in the experiment, EEGs are measured through the framework proposed when brainwaves are applied to a BCI contents. This framework collects user's brainwaves two times per second to analyze difference of brainwave, for a total of 90 times passing through the brainwave receiving stage. Figure 6 shows the diagram of measured brainwaves.

From collected values, we can found that many parts of user's brainwave values are below 60 . Dividing measured values, from 0 to 100 , into 10

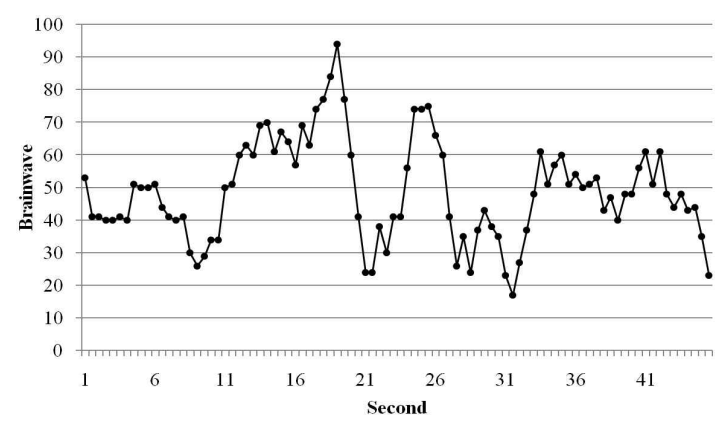

Fig. 6. User's collected brainwaves

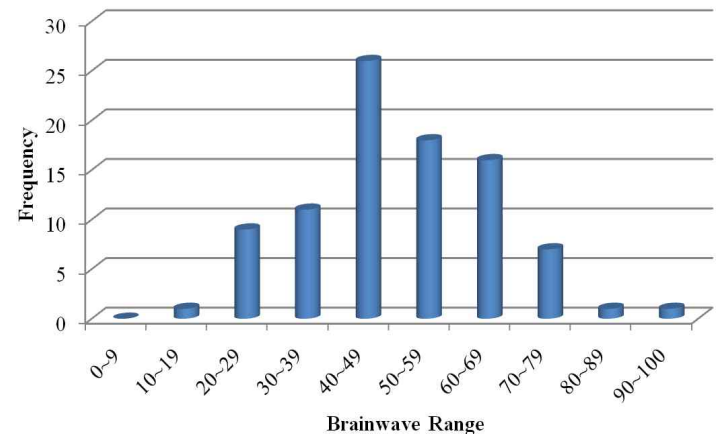

Fig. 7. Each frequency of ranges

sections and calculating occurrence frequency by section results as follows in Figure 7.

The highest brainwave frequency is shown in the section between 40 and 49 as 27 times. As shown in the distribution of brainwave values, most brainwaves are in the section between 40 and 69 , and the frequency toward both ends becomes lower. This distribution is utilized in user's brainwave normalization.

In the brainwave adjustment stage, measured brainwave values shifts by adding 10 , so that the section corresponding to the average is shifted to the higher position of the entire range.

Applying the result of normalization and adjustment to a BCI game is as shown in Figure 8. Figure 8(a) shows the measured values of concentration brainwaves that occurred through the brainwave receiving stage. Compared to Figure 6, it shows higher values, which means that the user is concentrating more.

Figure 8(b) shows the values that a user's measured brainwaves are applied to the brainwave normalization stage. We can found that brainwave values are adjusted up or down in some sections; the brainwaves after 3rd measurement show a higher value than the measured brainwaves in the brainwave collection step, while the brainwave values measured in the first time and second time are converted to lower values. In Figure 6, as having a brainwave above 50 is rare to the user, the section having brainwaves above 50 has been ad- 


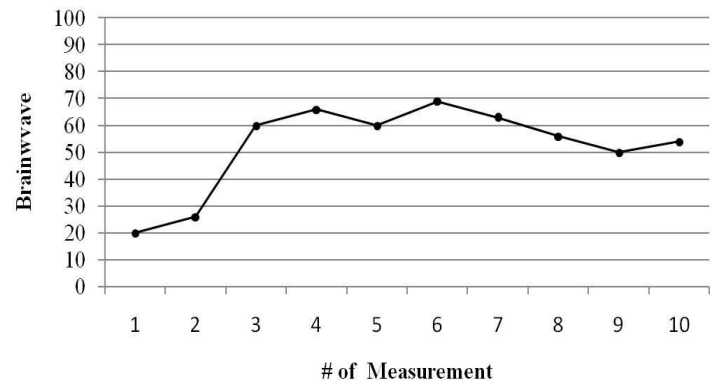

(a) Measured brainwaves

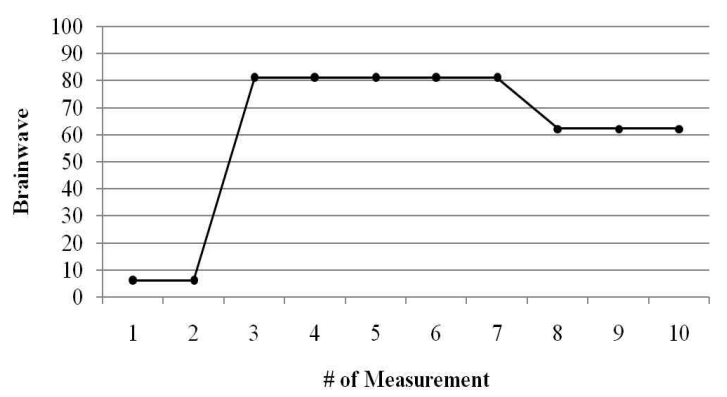

(b) Normalized brainwaves

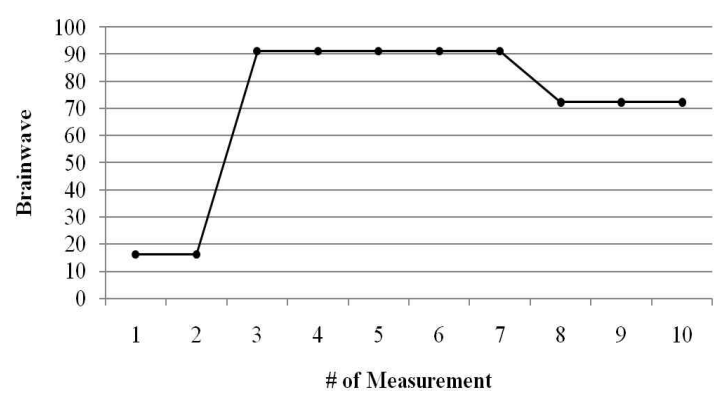

(c) Adjusted brainwaves

Fig. 8. Brainwave normalization and adjustment

justed up. On the other hand, where the user has a value of less than 20, it has been adjusted down.

Figure $8(\mathrm{c})$ is the adjusted diagram of brainwaves by 10 in the brainwave adjustment stage. The brainwaves that passed through the brainwave receiving stage and normalization stage are adjusted up.

The brainwaves that passed through all the stages are eventually transferred to Hello Neuron. Through this experiment, the brainwaves measured through brainwave receiving stage are adjusted by passing through the normalization stage and brainwave adjustment stage.

\section{CONCLUSION}

In this paper, the BCI framework for BCI contents to process brainwaves in real-time is proposed. This framework is composed of the brainwave receiving stage, brainwave normalization stage, and brainwave adjustment stage. The role of the brainwave receiving stage is to receive signals from various brainwave measuring devices and transfer them to the next stage. The brainwave normalization stage converts the signals that vary by person to a specific form of values. Finally, the brainwave adjustment stage provides the function to manually adjust each user's brainwaves.

In the experiment, the operation process by applying the proposed framework to a BCI game is presented. EEG signals from a user are processed by passing through the brainwave receiving stage, brainwave normalization stage, and brainwave adjustment stage. Through the experiment, it is shown that the EEG signal is corrected when it is applied to BCI contents according to the tendency of the user's brainwaves. The converting process of brainwaves having regular distribution would result in easy game development by using brainwaves. Therefore its process also reduce the required development time as well as have a cost saving effect.

This paper proposed the process of EEG signals. Another study for the automation tool to apply EEG signals to a game is planned to progress in the future. Because various configurations are required in order to apply the results to a game, if the study regarding an automation tool progresses, it would allow any BCI contents developer who is ignorant about brainwaves to conveniently develop BCI contents using brainwaves.

\section{ACKNOWLEDGEMENT}

This paper is presented by summarizing part of 
the research, "A Development of Serious Game Technology using Brain Computer Interface” that was performed by Dongguk University and was supported by Korea Creative Content Agency (KOCCA) in the Culture Technology(CT) Research \& Developement Program 2009.

\section{REFERENCE}

[1] S. I. Shin, J. H. Cho, and M. N. Kim, "Proposition for 4 Channel Frontal Lobe Electrode Configuration and Study on EOG Removal from Measure EEG." Journal of Korea Multimedia Society, Vol 6, No.1, pp.167-175, 2003.

[2] I. Park and B. Park "Brain Function Optimization Research using Neurofeedback," Kjscience, 2003.

[3] Emotiv, http://www.emotiv.com/

[4] A. Lécuyer, F. Lotte, R. B. Reilly, R. Leeb, M. Hirose, and M. Slater, "Brain-Computer Interfaces, Virtual Reality, and Videogames," Computer, Vol.41, Issue 10, pp.66-72, 2008.

[5] P. A. Pour, T. Gulrez, O. AlZoubi, G. Gargiulo, and R. A. Calvo, "Brain-Computer Interface: Next Generation thought Controlled Distributed Video Game Development Platform," IEEE Symposium On Computational Intelligence and Games 2008 (CIG '08), pp.251-257, 2008.

[6] A. Nijholt, B. Reuderink, and D. O. Bos, "Turning Shortcomings into Challenges: BrainComputer Interfaces for Games," Entertainment Computing, Vol.1, No.2, pp. 85-94, 2009.

[7] A. Finke, A. Lenhardt, and H. Ritter, "The MindGame: A P300-based Braincomputer Interface Game," Neural Networks, Vol.22, Issue 9, pp. 1329-1333, 2009.
[8] E. C. Lalor, S. P. Kelly, C. Finucane, R. Burke, R. Smith, R. B. Reilly, and G. McDarby, "Steady-State VEP-Based Brain Computer Interface Control in an Immersive 3-D Gaming Environment," EURASIP Journal of Applied Signal Processing, Vol.19, pp. 31563164, 2005.

[9] P. Martinez, H. Bakardjian, and A. Cichocki, "Fully Online Multi-Command Brain Computer Interface with Visual NeurofeedBack using SSVEP Paradigm," Computational Intelligence and Neuroscience, Vol.1, No.3, 2007.

[10] B. Shim, S. Lee, and J. Shin, "Implementation of a 3-Dimensional Game for Developing Balanced Brainwave," Conference on SERA 2007. 5th ACIS Intern., pp. 751-758, 2007.

[11] K. Müller, M. Krauledat, G. Dornhege, G. Curio, and B. Blankertz, "Machine Learning Techniques for Braincomputer Interfaces," Biomed. Tech, Vol.49, No.1, pp. 11-22, 2004.

[12] A. E. Selim, M. A. Wahed, and Y. M. Kadah, "Machine Learning Methodologies in P300 Speller Brain-Computer Interface Systems," Radio Science Conference 2009, NRSC 2009, National, pp. 1-9, 2009.

[13] Y. Li and C. Guan "A Semi-Supervised SVM Learning Algorithm for Joint Feature Extraction and Classification in Brain Computer Interfaces," 28th Annual International Conference of the IEEE Digital Object Identifier, pp. 2570-2573, 2006.

[14] G. Schalk, P. Brunner, L. A. Gerhardt, H. Bischof, and J. R. Wolpaw, "Brain - Computer Interfaces (BCIs): Detection Instead of Classification," Journal of Neuroscience Methods, Vol.167, Issue 1, pp. 51-62, 2008.

[15] Neurosky, http://www.neurosky.com/ 


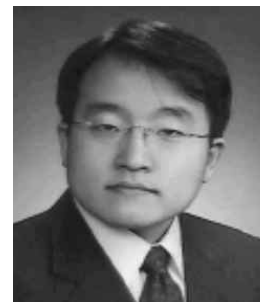

Sung, Yunsick

1997. 3 2004. 8 Division of Electrical and Computer Engineering, Pusan $\mathrm{Na}^{-}$ tional University (BS)

2004. 9 2006. 8 Computer Engineering, Dongguk University (MS)

2008. 3 present Game Engineering, Dongguk University ( $\mathrm{Ph} . \mathrm{D}$ candidate)

2006. 8 2009. 2 Samsung Electronics Company (Researcher)

Research Interests : Brain-computer Interface, Programming by demonstration, Ubiquitous $\mathrm{Com}^{-}$ puting and Reinforcement learning.

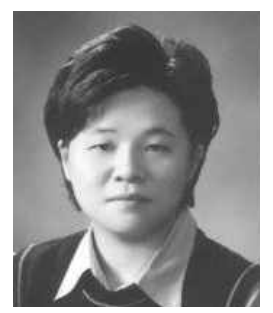

\section{Cho, Kyungeun}

1989. 3 1993. 2 Computer Science, Dongguk University (BS)

1993. 3 1995. 2 Computer Engineering, Dongguk University (MS)

1995. 3 2001. 8 Computer Engineering, Dongguk University (Ph.D)

2002. 3 2003. 2 Dept. of Digital Media, Fulltime Lecturer, Anyang University

2003. 3 2003. 9 Dept. of Game Engineering, Fulltime Lecturer, Youngsan University

2003. 9 2005. 8 Dept. of Computer Multimedia Engineering, Fulltime Lecturer, Dongguk University

2005. 9 2009. 8 Dept. of Game \& Multimedia Engineering, Assistant Professor, Dongguk University.

2009. 9 present Dept. of Multimedia Engineering, Associate Professor, Dongguk University.

Research Interests : Artificial Intelligence for Games, Game Algorithm, Computer Vision.

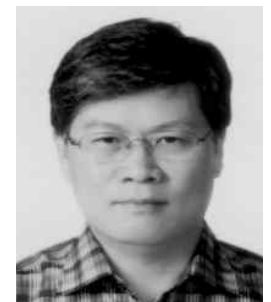

Um, Kyhyun

1971. 3 1975. 2 Dept. of Applied Mathematics, Engineering College, Seoul National University (BS)

1975. 3 1977. 2 Dept. of Computer Science, Korea $\mathrm{Ad}^{-}$ vanced Institute of $\mathrm{Sci}^{-}$ ence and Technology (MS)

1986. 3 1994. 2 Dept. of Computer Engineering, Graduate School, Seoul National University (Ph.D)

1978. 3 2006. 6 Dept. of Computer and Multimedia Engineering, Full Professor, Dongguk University

2006. $7 \sim$ present Dept. of Game and Multimedia Engineering, Full Professor, Dongguk University

2001. 3 2003. 2 College of Information and Industrial Engineering, Dean, Dongguk University

1995. 3 1999. 2 Information Management Institute, chief director, Dongguk University

1998. 8 2000. 7 Korea Information Science Society, SIGDB Chair

1999. 4 2005. 4 Int. Conf. on Database Systems for Advanced Applications (DASFAA) Steering Committee member

2007. 1 present Korean Multimedia Society, President

2004. 1 present Korean Game Society, Consulting member

Research Interests : Game Systems, Multimedia Applications 